\title{
El blog de aula y el WhatsApp cherramientas útiles para la comunicación entre maestras y familias?
}

\author{
Soledad García-Gómez; solgar@us.es \\ Universidad de Sevilla (España) \\ Mónica López-Gil; monica.maria@uca.es \\ Universidad de Cádiz (España)
}

Classroom Blog and WhatsApp, useful tools for teachers and families communication?

\section{Resumen}

La literatura específica muestra que hay dos herramientas tecnológicas con gran potencial para enriquecer la comunicación entre maestras y familias: los blogs de aula y el WhatsApp. El objetivo del estudio descriptivo ha sido explorar si estas herramientas son utilizadas para ampliar la comunicación entre ambos colectivos. Los datos se han recogido mediante entrevistas estructuradas a maestras de Educación Infantil. Tras categorizar los datos se han cuantificado y sometido a análisis del contenido textual. Resultados. En torno al $80 \%$ de las maestras no cuenta con blog de aula. Las razones primordiales son: la falta de tiempo y los problemas técnicos. Quienes sí lo tienen lo utilizan preferentemente como exposición de lo que ocurre en las aulas. El uso del WhatsApp tampoco es mayoritario, es rechazado con frecuencia por los malentendidos que suele producir. Las maestras que sí lo utilizan lo hacen a través de la madre delegada de clase. Se evidencia que hay problemas vinculados al uso de ciertas TIC que han de ser conocidos para tratar de paliarlos.

Palabras clave: Educación infantil, familias, comunicación, blogs, WhatsApp

\begin{abstract}
The state of the art evidences that communication between teachers and families will improve by using these two technological tools: classroom blogs and WhatsApp. The aim of this descriptive study has been to explore if these tools are nowadays really being use to extend communication. Data have been collected through structured interviews to preschool teachers. We have categorized and quantified them, and we have also done a textual content analysis. Results. Around $80 \%$ of teachers do not have a classroom blog. Main reasons for this scarce use are: lack of time and technical problems. Teachers who edit blogs consider they are very useful to show what is going on in the class. We have also found that WhatsApp is not frequently used by teachers because they try to avoid misunderstandings. They use this application only with the mother that represents the other families. It is obvious that there are some problems about the uses of ICT that should be known in order to solve them.
\end{abstract}

Keywords: Preschool Education, families, communication, blogs, WhatsApp 


\section{INTRODUCCIÓN}

La sociedad del conocimiento y la sociedad de la información suponen un desarrollo tecnológico que se caracteriza por su vertiginosidad. Es continua la creación de dispositivos, programas informáticos, plataformas, aplicaciones, etc. Es tal la actividad que, con frecuencia, su periodo de vigencia es muy breve, al ser mejorados y/o desbancados con gran rapidez. El desembarco de las tecnologías en la sociedad -también en el ámbito educativo- plantea situaciones inéditas, que merecen ser objeto de estudio (Pérez, Mercado, Martínez, Mena y Partida, 2018). Las denominadas Tecnologías de la Información y la Comunicación (TIC) son ensalzadas en la literatura pedagógica como recursos que facilitan la enseñanza, que promueven aprendizajes, que desarrollan competencias, que agilizan la burocracia de los centros escolares y que tienen un gran potencial para mejorar las relaciones familia-escuela. Disponemos ya de un elenco de investigaciones que visibilizan ventajas e inconvenientes de las nuevas modalidades de interlocución entre escuelas y familias (Ballesta y Cerezo, 2011; Beneyto-Seoane y Collet-Sabé, 2016; Freitas, Paredes y Sánchez-Antolín, 2019; Goodall, 2016; Macià y Garreta, 2018; Magdaleno y Llopis, 2014; Olmstead, 2013; Sánchez-Garrote y Cortada-Pujol, 2015).

La literatura sobre la participación de las familias del alumnado en las escuelas muestra que es en la etapa de educación infantil donde aquella es mayor. Madres, padres y tutores legales de este alumnado son quienes mantienen una mayor comunicación continuada con las maestras (Gomariz-Vicente, Hernández-Prados, García-Sanz y Parra-Martínez, 2017). Según el trabajo de Ruiz y Hernández (2018), centrado en el profesorado de educación infantil de Andalucía, algo más del 50\% de la muestra utiliza internet (móvil, correo electrónico...) para mantener contactos con las familias. En nuestro caso particular nos hemos preguntado si las maestras de Educación Infantil utilizan herramientas digitales como el blog de aula y el WhatsApp para ampliar y profundizar en las relaciones interpersonales que mantienen con las familias de su alumnado.

Bohórquez (2008) define el blog como "una página Web muy básica y sencilla donde el usuario puede colgar comentarios, artículos, fotografías, enlaces e incluso vídeos" (p. 1). Se considera una herramienta con gran potencial para favorecer la colaboración y la comunicación compartida entre escuelas y familias del alumnado (Solano, 2014). Insiste en ello Macià (2016), cuando afirma que antes no existía ningún otro instrumento que facilitase tanto el intercambio informativo entre las familias y el profesorado. También Pavón (2013) los presenta como una herramienta dinámica con virtudes diversas, entre las que se encuentra el facilitar esa relación. Asorey y Gil (2009) ensalzan su facilidad de uso, al destacar que son una herramienta muy sencilla, que cualquier persona puede crear y mantener.

En los últimos años, la aparición de dispositivos digitales ha afectado a los blogs (Lara, 2005). Thompson, Mazer y Grady (2015) sostienen que se está dejando de usar el ordenador en la comunicación familia-escuela, para centrarse únicamente en los teléfonos móviles. Esto concuerda con el uso mayoritario actual de este dispositivo respecto a otros de naturaleza digital (AIMC, 2019). 
En esta línea, una herramienta o recurso que ha venido cobrando relevancia en la comunicación entre las familias y los docentes es el WhatsApp, aplicación de mensajería instantánea multimedia y multiplataforma. El intercambio comunicativo sincrónico, su gratuidad y la posibilidad de compartir información en multiformato, así como la oportunidad de silenciar notificaciones, reenviar, salir del grupo, mantener muchos chats funcionando en paralelo, etc., la convierten en una de las redes sociales más utilizadas en contextos diversos (Vilches y Reche, 2019).

Sin embargo, como destacan Amara, Maceda, Bendella y Santos (2016), tras un momento de explosión, han aflorado ciertas reticencias hacia el uso del WhatsApp en el ámbito educativo: unas vinculadas a cuestiones técnicas y otras a problemas en las relaciones personales. Estas disfunciones ensombrecen sus virtudes. Cremades, Maqueda y Onieva (2016) recomiendan que "en el caso concreto de aplicaciones como WhatsApp, Messenger u otras similares, el profesorado debe cuestionarse sus posibles prejuicios negativos porque, más allá de los riesgos ya señalados, también puede ser un instrumento óptimo para aumentar la calidad de la educación" (p. 116).

Como es evidente, la irrupción de las TIC ha abierto nuevos cauces de interlocución y ha modificado rasgos de la comunicación tradicional entre ambos colectivos. Así pues, en el éxito o en el fracaso de estas vías de interrelación personal, intervienen distintas variables. Destacamos el peso de la tradición de otras formas de relación, la insuficiente alfabetización digital del profesorado y de las familias, y el papel crucial de los servicios técnicos. Es preciso atender con urgencia las necesidades formativas, tanto del profesorado -en activo y en formación- (Tejada y Pozos, 2018), como de las familias, para que las TIC se conviertan en herramientas provechosas para la interacción (GonzálezFernández, Ramírez-García y Salcines, 2018).

Desconocemos por el momento si las promociones de estudiantes que utilizan estas herramientas en su formación inicial (Molina, Valenciano y Valencia-Peris, 2015; Ruiz, Seva y Seva, 2016), serán más proclives a incorporarlas en su futuro desempeño como docentes. Algún trabajo reciente así lo vislumbra (Muñoz-Carril, González-Sanmamed y Fuentes-Abeledo, 2020).

\section{MÉTODOS}

\section{Objetivo y enfoque metodológico}

El objetivo del estudio ha sido explorar las prácticas cotidianas de comunicación de maestras de Educación Infantil con las familias de su alumnado. Especialmente el uso del blog de clase y del WhatsApp. Para este propósito hemos optado por un enfoque metodológico cualitativo centrado en la exploración a través de la descripción (Rodríguez, Gil y García, 1996). Para ello se ha optado por recabar los testimonios directos de las maestras (al ser un porcentaje tan elevado de mujeres optamos por el femenino como genérico del grupo) a través de una técnica de encuesta, como es la entrevista estructurada (Kvale, 2011). 


\section{Participantes}

Las personas informantes han sido docentes del segundo ciclo de educación infantil. Se ha realizado un muestreo de conveniencia, hasta alcanzar el punto de saturación de los datos. Finalmente han participado 72 docentes (68 maestras y 4 maestros) adscritos a colegios de Sevilla (capital y provincia). El $86 \%$ trabajaba en colegios públicos, mientras el 14\% restante lo hacía en centros concertados. La horquilla de años ejerciendo es bastante amplia, como se puede apreciar en la Tabla 1. Destacamos que casi el 70\% contaba con más de 10 años de experiencia.

Tabla 1. Porcentajes de rangos de años de experiencia docente

\begin{tabular}{|c|c|}
\hline Rangos de años de experiencia docente & Porcentajes \\
\hline $1-10$ años & $32 \%$ \\
\hline $11-20$ años & $37 \%$ \\
\hline $21-30$ años & $24 \%$ \\
\hline $31-36$ años & $7 \%$ \\
\hline
\end{tabular}

Técnica de recogida de datos

Como hemos anunciado, la técnica seleccionada para la recogida de datos fue la entrevista estructurada. La mayoría de las entrevistas se grabaron en audio en los propios centros educativos, datando del último trimestre del curso 2016/17. El guion incluía preguntas relativas a las dimensiones que se muestran a continuación, identificadas en la literatura como principales vías de comunicación entre las maestras y las familias (Maciá, 2019). Las preguntas eran del tipo: ¿utilizas la agenda escolar para comunicarte con las familias?, ¿tienes blog de aula?, ¿por qué no?, ¿qué te aporta?, etc.

a) Uso de la agenda escolar y de las circulares informativas.

b) Uso de la plataforma institucional del colegio y/o de la administración.

c) Encuentros informales a la entrada y salida del colegio.

d) Blog de clase.

e) Grupos de WhatsApp.

\section{Técnica de análisis de datos}

Como indican Osses, Sánchez e Ibáñez (2018), "el análisis cualitativo de los datos es el proceso no matemático de interpretación, llevado a cabo con el propósito de descubrir conceptos y relaciones y de organizarlos en esquemas teóricos explicativos" (p. 121). Para ello, la técnica de análisis de datos empleada ha sido el análisis de contenido tras seguir las fases de reducción, disposición y transformación. Así, después de transcribir las entrevistas se ha procedido a la lectura del contenido en reiteradas ocasiones. Teniendo en cuenta tanto las dimensiones que estructuraban el instrumento de recogida de datos, como los propios datos obtenidos, elaboramos un sistema de 
categorías de carácter inductivo. En la Tabla 2 se explicitan solo las categorías y subcategorías vinculadas a los datos utilizados en la elaboración de este artículo (corresponden a las dimensiones d y e).

Tabla 2. Sistema de categorías y subcategorías

\begin{tabular}{|l|l|}
\hline Categorías & Subcategorías (códigos) \\
\hline \multirow{4}{*}{ Blog de aula } & * No blog de aula (NOB) \\
& * No blog de aula ahora, antes sí (NBA) \\
& * Blog de aula con participación familias (BAP) \\
& * Blog de aula como repositorio de imágenes y recursos (BAR) \\
& * Blog de centro (BCE) \\
\hline \multirow{3}{*}{ Grupo de WhatsApp } & * No grupo de WhatsApp con familias del alumnado (NGW) \\
& * Sí grupo de WhatsApp (SGW) \\
& * Uso de WhatsApp con madre delegada de curso (WMD) \\
& * Posicionamiento favorable al uso de medios tecnológicos (TSI) \\
& * Rechazo al uso de medios tecnológicos (TNO) \\
\hline
\end{tabular}

Tras el proceso de identificación de las unidades de información procedimos a su codificación. Se ha utilizado un doble código: uno relativo a la subcategoría y otro relativo a la persona entrevistada. En este caso, los códigos permiten saber el sexo $(\mathrm{A} / \mathrm{O})$, si el centro es público o concertado $(\mathrm{P} / \mathrm{C})$ y su número de identificación (al respetarse el anonimato). Por ejemplo: una maestra que trabaja en un centro público, cuyo número de orden de la entrevista es 34, le corresponde el código AP34. A continuación, al presentar los resultados que incluyen fragmentos textuales de las entrevistas, solo indicaremos este tipo de código, pues el relativo a la temática está implícito en la exposición.

Los datos han sido contrastados y comparados a fin de identificar patrones representativos de los usos que las participantes hacen de estas herramientas. El fruto de este proceso es la presentación de resultados, que conducirá finalmente a la elaboración de conclusiones.

\section{RESULTADOS}

\section{Tecnologías ¿sí o no?}

En relación con el uso de las tecnologías para comunicarse con las familias del alumnado hemos identificado dos perfiles distintos. De una parte, dos de las maestras, con más de veinticinco años de ejercicio profesional, explicitaban claramente su beneplácito con las tecnologías para avanzar en el terreno de las relaciones con las familias: 
“Cada vez usamos menos los papeles informativos. Antes prácticamente era el único medio para comunicar, para comunicarnos, pero ahora las nuevas tecnologías nos han facilitado estrategias que son mucho más rápidas, más eficaces y más modernas" (AP23)

"La tecnología ha dado un gran avance en cuanto a la comunicación de profes, padres y alumnos" (AP66).

De otra parte estaban las maestras más reticentes con su empleo. Una de ellas, con 16 años de desempeño docente, afirmaba: "de toda la vida hemos dado papelitos escritos y así ha llegado la información ¿vale?" (AP58). No mostraba interés por usar las nuevas herramientas disponibles, como tampoco lo hacía otra maestra que apuesta por la comunicación personal, resaltando el valor de las relaciones sociales:

"Mis alumnos sin Internet aprenden, leen, escriben, juegan, respetan normas ¿por qué? Porque lo importante es lo social, la comunicación y la relación social, y eso no lo da ni el blog, ni Internet, ni los teléfonos... por mucho que nos comuniquemos con ellos" (AP61).

\section{Uso del blog de aula y/o del WhatsApp}

Aunque el estudio ha girado en torno a varias vías de comunicación, en este artículo sólo nos ocupamos de sus posicionamientos en relación al uso del blog de aula y de los grupos de WhatsApp. La Tabla 3 muestra los principales resultados a nivel porcentual.

Tabla 3. Uso de blogs y grupos de WhatsApp

\begin{tabular}{|l|l|}
\hline Dispone de blog de aula & \\
\hline Sí & $>17 \%$ \\
\hline No & $>80 \%$ (17\% lo abandonó; $16 \%$ tiene blog en el colegio) \\
\hline Participa grupo WhatsApp & $<8 \%$ \\
\hline Sí & $50 \%$ \\
\hline No & $>40 \%$ \\
\hline $\begin{array}{l}\text { A través de madre } \\
\text { delegada de la clase }\end{array}$ & \\
\hline
\end{tabular}

La Figura 1 aporta más datos que son descritos en los siguientes apartados. 


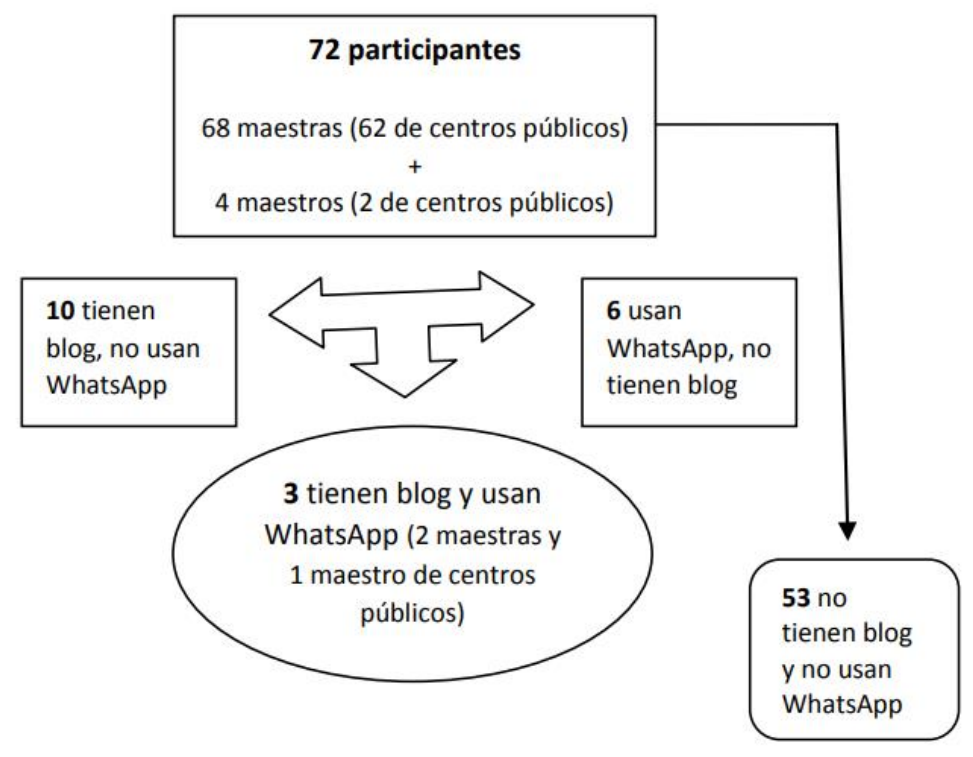

Figura 1. Distribución de docentes según si tienen blog de aula y/o usan WhatsApp

Como se puede apreciar, 53 docentes no tienen blog de aula y tampoco usan WhatsApp. Hay 10 que tienen blog frente a 6 que usan WhatsApp, mientras sólo 3 emplean ambas herramientas. Estas tres personas cuentan con una dilatada experiencia como docentes: una maestra con 16 años, un maestro con 30 y una maestra con 29. Es evidente que son una clara minoría del total y que no son perfiles de docentes jóvenes que, previsiblemente, podría considerarse que están más familiarizados con las TIC.

Una de las maestras justifica el uso del blog vinculado al trabajo por proyectos que realiza en el aula. Utiliza el teléfono móvil tanto para editar el blog como para comunicarse a través del WhatsApp.

"No requiere mucho tiempo ya que tengo la aplicación en el móvil y directamente hago las fotos y las subo y pongo un texto pequeñito (...) A mí me resulta muy cómodo si tengo que mandar algo para todos los padres, me es mucho más rápido poner para todos un WhatsApp que escribir un papel en un ordenador, imprimirlo, sacarle fotocopias, metérselo a cada uno en la mochila... a mí me resulta muy cómodo" (AP26).

El maestro que tiene blog y usa el WhatsApp cuenta con 30 años de ejercicio profesional. A pesar de llevar "muchos" años con blog, sus palabras vislumbran que éste está siendo relegado: "Últimamente el WhatsApp está desplazando al blog" (OP28). 
Blog de aula ¿sí o no?

Como hemos indicado, más del $80 \%$ de las maestras no tiene un blog de aula. Solo un $17 \%$ declara tenerlo, habiendo otro $17 \%$ aproximadamente que lo tuvo y lo ha dejado. Un 16\% comenta que no tiene blog de aula, pero sí dispone de blog en el colegio. Ante la contundencia de los números, que muestran de forma explícita que el blog de aula no es en la actualidad una herramienta muy usada por parte del profesorado de Educación Infantil, exploramos las justificaciones aportadas.

El principal argumento a favor es que se trata de una potente fuente de información para las familias. Les resulta útil para presentar las tareas que se hacen en clase, para mostrar fotos, anunciar eventos, etc. Es un uso a modo de diario textual y gráfico de las clases, convirtiéndose en ocasiones en un escaparate de la vida del aula.

"Me parecen muy útiles porque permiten que siga habiendo una comunicación más allá del horario escolar y, sobre todo, permiten que las familias vean el desarrollo diario de los niños que, muchas veces, si no es así, no se ve lo que estamos haciendo, ni por dónde vamos, ni lo que estamos explicando, ni nada " (AC38).

Apenas se hace mención a la participación de las familias en los blogs, más allá de hacerlo como meros consumidores de un contenido establecido por las maestras. Destacamos las palabras de un maestro que sí obtiene retroalimentación.

"Los padres participan, tenemos muchos comentarios, nuestro blog es muy interactivo" (OP70).

Las principales razones esgrimidas para no disponer de blog de aula son variadas. De una parte, la falta de tiempo, pues se entiende como una tarea añadida a todas las demás. En ocasiones se vincula con la asunción de nuevas responsabilidades, como la coordinación del ciclo. Aducen que tienen gran carga de trabajo y que están desbordadas; de hecho, había quien lo tenía y lo ha abandonado. De otra parte, el insuficiente dominio de la herramienta, que hace que su uso sea complicado. Además, en ocasiones, se encuentran con problemas técnicos relativos a la red (lentitud, sobrecarga, desconexión), que les llevan a desistir de su uso.

"Hasta septiembre estaba actualizado, pero hemos tenido problemas con la Internet del centro..." (AP06).

Las condiciones laborales también pueden llegar a pesar para optar o no por el blog. Una de las maestras dice que tiene más dificultades por el hecho de ser interina. El cambio habitual de colegio le genera problemas a este respecto:

"... si la interinidad se producía por sustitución y el tiempo que podía trabajar en algunos centros era limitado, montar un blog para tenerlo que desmontar era complicado" (AP57). 
El nivel sociocultural de las familias del alumnado también lo consideran en algunos casos como una limitación para el manejo de este tipo de recursos.

"En mi entorno, como existen familias con un nivel cultural bajo, la conversación directa es la mejor" (AP33).

Al caracterizar a las personas entrevistadas planteamos que la gran mayoría eran maestras que trabajan en centros públicos. La singularidad está en los maestros y en quienes trabajan en centros concertados. Hacemos una breve referencia a la situación de los cuatro maestros varones: solo uno de ellos tiene blog desde hace varios años y otro está interesado en tenerlo. Más de la mitad de quienes trabajan en centros concertados no tiene blog personal; un tercio del total participa en el blog del centro.

\section{Participar en el grupo de WhatsApp con las familias ¿sí o no?}

Pasamos ahora a presentar qué ocurre con el uso del WhatsApp. Alrededor del 8\% de las maestras sí forma parte del grupo establecido con las familias de su alumnado. Expresan un rotundo rechazo a participar más del $50 \%$, mientras un $40 \%$ se comunica por esta vía solo con la madre delegada de clase, quien hace de intermediaria con las demás madres/padres/tutores legales.

Las cinco maestras y el maestro que se muestran a favor del uso del WhatsApp destacan de esta herramienta la rapidez, el fácil manejo, el ahorro de tiempo, la comodidad, etc. Apuestan por su uso, teniendo muy claro cuáles son sus virtudes en el ámbito escolar.

"Para mí es útil porque es una vía muy rápida para informar a las familias y que las familias te informen de cualquier cosa (...) A mí me va muy bien, no tengo ningún problema" (AP24).

"Nunca ha habido polémica con el WhatsApp, yo soy el administrador ... es una cosa fantástica" (OP28).

Hay diversidad de situaciones entre quienes lo utilizan para comunicarse con las familias a través de un intermediario (madre o padre delegado de clase). En algunos casos es una imposición del centro educativo, en otros es una demanda de las familias, o son las maestras quienes han optado por esta fórmula. En general hay interés por beneficiarse de sus bondades y bastante recelo por los problemas que pueda ocasionar.

"Dejé claro que respecto al WhatsApp que no quería críticas de ningún tipo, dije que la madre delegada que creó el grupo sería la responsable, porque se han dado casos en los que se han impreso conversaciones y se han llevado a juicio (...) para cosas muy específicas sí sirve, pero, incluso así, pienso que el WhatsApp es totalmente prescindible" (AP65).

Quienes se muestran rotundamente en contra del uso de esta herramienta suelen centrar sus críticas en los problemas de comunicación que se pueden generar, en los malentendidos, en los 
usos superfluos, en el papel secundario que pasa a ocupar el alumnado, en la invasión de la intimidad, en las continuas exigencias, etc.

"Yo, ni loca, al final son problemas y en vez de usarse para lo que se debería se convierte en decir lo que mi hijo ha comido hoy..." (AC35).

Alguna maestra comenta que no está de acuerdo con el uso de los grupos, pero sí lo utiliza para comunicarse con alguna familia en particular.

"De forma grupal no, pero de forma individual hay momentos en los que es necesario comunicarse con las familias y, como es tan rápido, los problemas se solucionan al momento" (AP32).

La mayoría del profesorado de los centros concertados no participa en grupos de WhatsApp con las familias.

\section{DISCUSIÓN Y CONCLUSIONES}

Los resultados muestran con claridad que, en el caso particular de los blogs y el WhatsApp, las maestras no suelen usar estas herramientas digitales de forma asidua para comunicarse con las familias de su alumnado. Se constata que, a pesar de la reconocida potencialidad comunicativa de ciertas TIC en el ámbito escolar, estas no siempre son utilizadas de forma generalizada (independientemente de la edad de las maestras). Este hallazgo está en la línea de los trabajos de Magdaleno y Llopis (2014) y de Garreta (2015), quien afirma que "en los centros de infantil y primaria no se está aprovechando lo suficiente el potencial de las nuevas tecnologías para que fluya la información y la comunicación entre familias y profesionales" (p. 83). Beneyto-Seoane y Collet-Sabé (2016) confirman que las tecnologías no están sirviendo para que los centros educativos construyan unas relaciones mejores con todas las familias.

El acceso rápido y fácil a numerosos blogs de aulas en la red no puede llevarnos a pensar que son un recurso generalizado en la actualidad. A pesar de las virtudes que les asocian autores como Ray (2006) y Asorey y Gil (2009), los resultados de este estudio muestran que muchas maestras de infantil no los utilizan. Algunas de las razones para no emplearlos son: atenderlos implica un tiempo del que no disponen, tienen carencias formativas y han de afrontar problemas de carácter técnico. Nos parece importante identificar estas limitaciones porque, de otra forma, pudiese parecer que no existen, por lo que no se reclamarían actuaciones para poder mitigarlas.

Quienes tienen blogs asumen en gran medida que estos son una ventana por donde las familias pueden asomarse al interior de las aulas. Funcionan a modo de tablón de anuncios, pero apenas se han constituido en vehículos de comunicación bidireccional. Por ello, entendemos que disponer de blog de aula no siempre implica que la comunicación entre la familia y la maestra sea rica y 
fluida; tampoco que las familias participen más en la vida del aula. Abelleira y Abelleira (2013), maestras ganadoras del Premio Francisco Giner de los Ríos por su blog InnovArte Educación Infantil, confirman:

La existencia de miles de blogs de aula de maestros concretos que, a modo de ventana, permiten que las actividades realizadas con su grupo sean primordialmente conocidas por los progenitores de sus alumnos, pero que su concreción tan particular los convierte en un diario o en un anecdotario digital (p. 13).

El blog, como ventana para asomarse al interior de las aulas, puede llevar a las familias a conocer de forma más inmediata, continuada y detallada la vida escolar, aumentando así su confianza en el quehacer de las maestras. En unos años en los que la profesión docente ha sufrido un empeoramiento de sus condiciones laborales, acompañadas de cierto desprestigio social, mostrar su práctica y sus logros a través de los blogs, bien podría ser una estrategia para obtener cierto reconocimiento.

Es indiscutible que el blog, además de procurar un acercamiento a las familias, es una valiosa herramienta para favorecer el desarrollo profesional. Es una modalidad de diario de clase que permite reflexionar sobre la práctica, compartir y debatir con otras personas, así como orientar la toma de decisiones. Se trata de un recurso para el aprendizaje, como han planteado Muñoz-Carril et al. (2020).

Los resultados han evidenciado que la participación de estas maestras en los grupos de WhatsApp con las familias es muy escasa. Se suele rechazar su uso, incluso de forma muy rotunda. Las ventajas que asocian al WhatsApp (uso rápido, económico, fácil y global) no son suficientes para neutralizar las limitaciones y/o problemas que le achacan. Entre ellos: uso abusivo, uso en horario inoportuno, intromisiones en la intimidad, conversaciones sobre temas ajenos a lo educativo, conflictos comunicativos, malas interpretaciones...

Cremades et al. (2016) reconocen también que esta aplicación cuenta tanto con virtudes como con inconvenientes, algunos de los cuales coinciden con los nombrados por las personas participantes en este estudio. Las maestras se muestran a favor de utilizar la aplicación para transmitir mensajes a modo de avisos y recordatorios a las familias, pero no para mantener ningún otro tipo de comunicación bidireccional. Se confirma de nuevo que las relaciones familia-escuela no están siendo favorecidas por el uso de esta herramienta.

Constatamos que los blogs y el WhatsApp se están limitando en gran medida a ser vehículos de transmisión unidireccional de informaciones diversas. Este hallazgo nos lleva a retomar el reto planteado hace casi una década por Aguilar y Leiva (2012):

La creciente necesidad de establecer cauces efectivos de comunicación entre familia y escuela nos permite reafirmar la realidad innegable de la potencialidad que ofrecen las TIC, 
como herramientas relevantes para promover el intercambio, la interacción y la colaboración de las familias en la construcción de una escuela más democrática y participativa. Esto requiere un enfoque comunitario que implique a la escuela, a las familias y cómo no a las instancias políticas y sociales (p. 16).

Una conclusión de este estudio es que disponer de más cantidad y diversidad de recursos tecnológicos no siempre se traduce en una relación familia-escuela más estrecha. La comunicación y la participación en el seno de las comunidades educativas no están directamente relacionadas con el uso de las TIC, aunque sí pueden verse facilitadas por éstas. Para atender este propósito es necesario ser conscientes de las desigualdades de acceso y apropiación de las TIC por parte de las familias. También de las lagunas formativas del profesorado para un uso didáctico, relacional y cooperativo de aquellas. Es deseable y urge ir dando pasos hacia el desarrollo de la competencia digital responsable y ética, tanto de las familias como del profesorado, al tiempo que se avanza en el uso pedagógico de las tecnologías (Céspedes y Ballesta, 2018; Fernández, Fernández y Rodríguez, 2018; González-Fernández et al. 2018; Macià y Garreta, 2018; Sancho, Alonso y Sánchez, 2018).

También es preciso que los centros educativos cuenten con otros profesionales que se ocupen de aspectos técnicos, que se facilite la inmersión del profesorado en los procesos formativos necesarios, que se flexibilicen y mejoren las condiciones laborales, así como que se aborden desde los centros los cauces de participación de las familias. Todo el peso de la incorporación de las TIC a la escuela no puede recaer en el profesorado. Tampoco las TIC pueden protagonizar todas las relaciones entre profesorado y familias. Más aun cuando se constata que no todas las familias tienen igual acceso a las tecnologías (Ballesta y Cerezo, 2011). Tenemos que ser cautos para no agudizar las diferencias de partida entre las familias, segregando a algunas de ellas.

Es por ello por lo que creemos fundamental que se reconsideren ciertos discursos y actuaciones, tanto a nivel de la formación inicial del profesorado como de la formación permanente (Kereluik, Mishra, Fahnoe y Terry, 2013; Escudero, González y Rodríguez, 2018; Tejada y Pozos, 2018). Además, habrá que revisar las disposiciones vinculadas a las condiciones laborales del profesorado en activo. Aparte de conocimientos técnicos sobre las herramientas, es preciso contar con modelos conceptuales vinculados a enfoques educativos democráticos e inclusivos. Competencia digital e inclusión social han de conformar un binomio bien avenido. Como señalan Cabero-Almenara y Ruiz-Palmero (2017), la incorporación de las TIC en el ámbito educativo debe estar planificada "por una parte para empoderar a las personas y facilitar su inclusión social y digital, tanto a la educación en particular como a la sociedad en general, y por otra, para favorecer el desarrollo de la persona" (p. 25).

Para finalizar, señalar que las principales limitaciones del estudio se refieren a la muestra y al instrumento de recogida de datos. En futuros trabajos se debería manejar una muestra más amplia y representativa. Sería deseable conocer también las opiniones de las familias. El instrumento debería ser más abierto para facilitar la profundización en las respuestas. Creemos que así se 
podría dar un paso más en el conocimiento y análisis del uso (y rechazo) de las TIC como medios de comunicación y participación en la relación familia-escuela.

\section{REFERENCIAS}

Abelleira, A. y Abelleira, I. (2013). InnovArte. Educación Infantil, una historia de vida profesional. Padres y Maestros, 353, 10-13. Recuperado de: https://revistas.comillas.edu/index.php/padresymaestros/article/view/1700

Aguilar, MạC. y Leiva, J.J. (2012). La participación de las familias en las escuelas TIC: Análisis y reflexiones educativas. Pixel-Bit, Revista de Medios y Educación, 40, 7-19. Recuperado de: https://recyt.fecyt.es/index.php/pixel/article/view/61431

AIMC (2019). Marco General de los Medios en España 2019. Recuperado de: https://www.aimc.es/a1mc-c0nt3nt/uploads/2019/01/marco19.pdf

Amara, S., Macedo, J., Bendella, F. \& Santos, A. (2016). Group Formation in Mobile Computer Supported Collaborative Learning Contexts: A Systematic Literature Review. Journal of Educational Technology \& Society, 19(2), 258-273. Recuperado de: http://www.jstor.org/stable/jeductechsoci.19.2.258

Asorey, E. y Gil, J. (2009). El placer de usar las Tic en las aulas de infantil. CEE. Participación educativa, 12, 110-119.

Ballesta, J. y Cerezo, Ma C. (2011). Familia y escuela ante la incorporación de las tecnologías de la información y la comunicación. Educación XX1, 14(2), 133-156. Recuperado de: http://www.redalyc.org/articulo.oa?id=70618742006

Beneyto-Seoane, M. y Collet-Sabé, J. (2016). Las relaciones digitales entre familias y escuela: análisis y propuestas. @tic. revista d'innovació educativa, 16, 1-9. Recuperado de: https://doi.org/10.7203/attic.16.6894

Bohórquez, E. (2008). El blog como recurso educativo. EDUTEC. Revista Electrónica de Tecnología Educativa, 26, 1-10. https://doi.org/10.21556/edutec.2008.26.468

Cabero-Almenara, J. y Ruiz-Palmero, J. (2017). Las Tecnologías de la Información y Comunicación para la inclusión: reformulando la brecha digital. IJERI. International Journal of Educational Research and Innovation, 9, 16-30. Recuperado de: https://www.upo.es/revistas/index.php/IJERI/article/view/2665 
Céspedes, R. y Ballesta, J. (2018). Acceso, uso y actitud de la tecnología en las escuelas de Educación Primaria de la Región de Murcia. Aula Abierta, 47(3), 355-364. https://doi.org/10.17811/rifie.47.3.2018.355-364

Cremades, R., Maqueda, E. y Onieva, J.L. (2016). Posibilidades didácticas de la escritura digital ubicua en la aplicación WhatsApp Messenger. Revista Electrónica de Estudios Transatlánticos de Literatura, 16, 106-120. Recuperado de: https://revistaseug.ugr.es/index.php/letral/article/view/4932/4741

Escudero, J. M., González, Mạ. T. y Rodríguez, Ma․ J. (2018). Los contenidos de la formación continuada del profesorado: ¿Qué docentes se están formando? Educación XX1, 21(1), 157180. https://doi: 10.5944/educXX1.15807

Fernández, F.J., Fernández, M.J. y Rodríguez, J.M. (2018). El proceso de integración y uso pedagógico de las TIC en los centros educativos madrileños. Educación XX1, 21(2), 395-416. https://doi.org/10.5944/educxx1.17907

Freitas, A; Paredes, J. y Sánchez-Antolín, P. (2019). Los espacios intermedios de la relación entre familia y escuela en contextos de inmersión tecnológica en Educación Primaria. Revista Latinoamericana de Tecnología Educativa, 18(1), 41-53. http://dx.medra.org/10.17398/1695-288X.18.1.41

Garreta, J. (2015). La comunicación familia-escuela en la Educación Infantil y Primaria. Revista de la Asociación de Sociología de la Educación, 8(1), 71-85.

Gomariz-Vicente, Mạa.; Hernández-Prados, Mạ A.; García-Sanz, M.P. y Parra-Martínez, J. (2017). Tejiendo puentes entre la escuela y la familia. El papel del profesorado. Bordón. Revista de Pedagogía, 69(2), 41-57. Recuperado de: https://doi.org/10.13042/Bordon.2016.49832

González-Fernández, N., Ramírez-García, A. y Salcines, I. (2018). Competencia mediática y necesidades de alfabetización audiovisual de docentes y familias españolas. Educación XX1, 21(2), 301-321. https://doi.org/10.5944/educXX1.16384

Goodall, J.S. (2016). Technology and school-home communication. International Journal of Pedagogies and Learning, 11(2), 118-131. http://dx.doi.org/10.1080/22040552.2 $\underline{016.1227252}$

Kereluik, K., Mishra, P., Fahnoe, Ch. \& Terry, L. (2013). What Knowledge Is of Most Worth: Teacher Knowledge for 21st Century Learning. Journal of Digital Learning in Teacher Education, 29(4), 127-140. Recuperado de: https://files.eric.ed.gov/fulltext/EJ1010753.pdf

Kvale, S. (2011). Las entrevistas en la investigación cualitativa. Madrid, España: Morata. 
Lara, T. (2005). Blogs para educar. Usos de los blogs en una pedagogía constructivista. TELOS. Cuadernos de Comunicación e Innovación, 5, 86-93. Recuperado de: http://unileon.pbworks.com/f/edublogs.pdf

Macià, M. (2016). La comunicación familia-escuela: el uso de las TIC en los centros de primaria. Revista Electrónica Interuniversitaria de Formación del Profesorado, 19(1), 73-83. Recuperado de: https://revistas.um.es/reifop/article/view/245841

Macià, M. (2019). Principales canales para la comunicación familia-escuela: análisis de necesidades y propuestas de mejora. Revista Complutense de Educación, 30(1), 147-165. http://dx.doi.org/10.5209/RCED.56034

Macià, M. y Garreta, J. (2018). Accesibilidad y alfabetización digital: barreras para la integración de las TIC en la comunicación familia/escuela. Revista de Investigación Educativa, 36(1), 239257. https://doi.org/10.6018/rie.36.1.290111

Magdaleno, A. y Llopis, MaA. (2014). La actitud docente y el grado de uso de las TIC en la comunicación familia-escuela: Un acercamiento al empleo de las TIC en las escuelas. Fòrum de Recerca, 19, 393-409. http://dx.doi.org/10.6035/ForumRecerca.2014.19.25

Molina, P., Valenciano, J. y Valencia-Peris, A. (2015). Los blogs como entornos virtuales de enseñanza y aprendizaje en educación superior. Revista Complutense de Educación, 26 (Especial), 15-31. https://doi.org/10.5209/rev RCED.2015.v26.43791

Muñoz-Carril, P; González-Sanmamed, M. y Fuentes-Abeledo, E. (2020). Use of blogs for prospective early childhood teachers. Educación XX1, 23(1), 247-273. https://doi.org/10.5944/educxx1.23768

Olmstead, C. (2013). Using Technology to Increase Parent Involvement in Schools. TechTrends, 57(6), 28-37. https://doi.org/10.1007/s11528-013-0699-0

Osses, S., Sánchez, I. y Ibáñez, F. (2018). Investigación cualitativa en educación. Hacia la generación de teoría a través del proceso analítico. Estudios Pedagógicos, 32(1), 119-133. http://dx.doi.org/10.4067/s0718-07052006000100007

Pavón, F. (2013). La introducción de las TIC en el currículum y en la organización escolar de la educación infantil y primaria. En M.C. Martínez, Buenas prácticas educativas en el uso de las TIC (pp. 11-50). Jaén: Joxman Editores. Recuperado de: http://hdl.handle.net/10498/16431

Pérez, R., Martínez, M., Mena, E. y Partida, J.A. (2018). La sociedad del conocimiento y la sociedad de la información como la piedra angular en la innovación tecnológica educativa. RIDE. 
Revista Iberoamericana de Investigación y Desarrollo Educativo, 8(16), 847-870. http://dx.doi.org/10.23913/ride.v8i16.371

Ray, J. (2006). Welcome to the Blogosphere. Kappa Delta Pi Record, 42(4), 175-177. https://doi.org/10.1080/00228958.2006.10518024

Rodríguez, G.; Gil, J. y García, E. (1996). Metodología de la investigación cualitativa. Archidona: Aljibe.

Ruiz, M.C. y Hernández, V.M. (2018). La incorporación y uso de las TIC en educación infantil. Un estudio sobre la infraestructura, la metodología didáctica y la formación del profesorado en Andalucía. Píxel-Bit. Revista de Medios y Educación, 52, 81-96. http://dx.doi.org/10.12795/pixelbit.2018.i52.06

Ruiz, D., Seva, P. y Seva, S. (2016). Relación vía Whatsapp entre padres y maestros/as. Perspectiva del futuro docente. En R. Roig-Vila, Tecnología, innovación e investigación en los procesos de enseñanza-aprendizaje (pp. 1233-1240). Barcelona, España: Octaedro.

Sánchez-Garrote, I. y Cortada-Pujol, M. (2015). Recursos digitales en la relación familia y escuela en la etapa 0-3. Cultura y Educación, 27(1), 221-233. http://dx.doi.org/10.1080/11356405.2015.1006851

Sancho, J., Alonso, C. y Sánchez, J.A. (2018). Miradas retro-prospectivas sobre las Tecnologías Educativas. Educatio Siglo XXI, 36(2), 209-228. https://doi.org/10.6018/j/333051

Solano, T. (2014). Blogs. En F. Trujillo (coord.), Artefactos digitales. Una escuela digital para la educación de hoy (pp. 40-43). Barcelona, España: Graó.

Tejada, J. y Pozos, K.V. (2018). Nuevos escenarios y competencias digitales docentes: hacia la profesionalización docente con TIC. Profesorado. Revista de Currículum y Formación del Profesorado, 22(1), 25-51. Recuperado de: https://recyt.fecyt.es/index.php/profesorado/article/view/63620

Thompson, B.C., Mazer, J.P. \& Grady, E.F. (2015). The Changing Nature of Parent-Teacher Communication: Mode Selection in the Smartphone Era. Communication Education, 64(2), 187-207. https://doi.org/10.1080/03634523.2015.1014382

Vilches, M.J. y Reche, E. (2019). Limitaciones de WhatsApp para la realización de actividades colaborativas en la universidad. RIED. Revista Iberoamericana de Educación a Distancia, 22(2), 57-77. https://doi.org/10.5944/ried.22.2.23741 
Para citar este artículo:

García-Gómez, S., y López-Gil, M. (2020). El blog de aula y el WhatsApp ¿herramientas útiles para la comunicación entre maestras y familias?. Edutec. Revista Electrónica De Tecnología Educativa, (72), 17-53. https://doi.org/10.21556/edutec.2020.72.1613 